\title{
In vivo effects of dietary quercetin and quercetin-rich red onion extract on skeletal muscle mitochondria, metabolism, and insulin sensitivity
}

\author{
T. M. Henagan - W. T. Cefalu • D. M. Ribnicky $\cdot$ R. C. Noland $\cdot$ \\ K. Dunville · W. W. Campbell • L. K. Stewart • L. A. Forney • \\ T. W. Gettys $\cdot$ J. S. Chang $\cdot$ C. D. Morrison
}

Received: 2 August 2014/Accepted: 4 December 2014/Published online: 27 December 2014

(C) Springer-Verlag Berlin Heidelberg 2014

\begin{abstract}
Red onions and low doses of the flavonoid, quercetin, increase insulin sensitivity and improve glucose tolerance. We hypothesized that dietary supplementation with red onion extract (RO) would attenuate high fat diet (HFD)-induced obesity and insulin resistance similar to quercetin supplementation by increasing energy expenditure through a mechanism involving skeletal muscle mitochondrial adaptations. To test this hypothesis, C57BL/6J mice were randomized into four groups and fed either a low fat diet (LF), HFD (HF), HFD + quercetin $(\mathrm{HF}+\mathrm{Q})$, or $\mathrm{HFD}+\mathrm{RO}(\mathrm{HF}+\mathrm{RO})$ for 9 weeks. Food consumption and body weight and composition were measured weekly. Insulin sensitivity was assessed by insulin and glucose tolerance tests. Energy expenditure and physical activity were measured by indirect calorimetry. Skeletal muscle incomplete beta oxidation, mitochondrial number, and mtDNA-encoded gene expression were measured. Quercetin and RO supplementation decreased HFD-induced fat mass accumulation and insulin resistance (measured by insulin tolerance test) and increased energy expenditure;
\end{abstract}

T. M. Henagan $(\bowtie) \cdot$ K. Dunville $\cdot$ W. W. Campbell Department of Nutrition Science, Purdue University,

West Lafayette, IN, USA

e-mail: thenagan@purdue.edu

W. T. Cefalu

John S. McIlhenny Botanical Research, Pennington Biomedical

Research Center, Baton Rouge, LA, USA

D. M. Ribnicky

Department of Plant Biology and Pathology, Rutgers University, New Brunswick, NJ, USA

R. C. Noland

Skeletal Muscle Metabolism, Pennington Biomedical Research

Center, Baton Rouge, LA, USA however, only $\mathrm{HF}+\mathrm{Q}$ showed an increase in physical activity levels. Although quercetin and RO similarly increased skeletal muscle mitochondrial number and decreased incomplete beta oxidation, establishing mitochondrial function similar to that seen in LF, only HF $+\mathrm{Q}$ exhibited consistently lower mRNA levels of mtDNAencoded genes necessary for complexes IV and V compared to LF. Quercetin- and RO-induced improvements in adiposity, insulin resistance, and energy expenditure occur through differential mechanisms, with quercetin-but not RO-induced energy expenditure being related to increases in physical activity. While both treatments improved skeletal muscle mitochondrial number and function, mtDNAencoded transcript levels suggest that the antiobesogenic, insulin-sensitizing effects of purified quercetin aglycone, and RO may occur through differential mechanisms.

Keywords Quercetin - Insulin resistance - Obesity · Mitochondrial number · Skeletal muscle $\cdot$ Energy expenditure
L. K. Stewart

Rocky Mountain Cancer Rehabilitation Institute, University of Northern Colorado, Greely, CO, USA

L. A. Forney · T. W. Gettys · J. S. Chang

Laboratory of Nutrient Sensing and Adipocyte Signaling,

Pennington Biomedical Research Center, Baton Rouge,

LA, USA

C. D. Morrison

Laboratory of Neurosignaling, Pennington Biomedical Research

Center, Baton Rouge, LA, USA 


\section{Introduction}

High fat diet (HFD)-induced obesity is associated with the development of insulin resistance mediated partially by decreased mitochondrial number and promotion of mitochondrial dysfunction (Jia et al. 2014; Lowell and Shulman 2005; Szendroedi et al. 2012). Purified bioflavonoid supplementation in conjunction with HFD effectively promotes mitochondrial biogenesis and improves mitochondrial function (Carrasco-Pozo et al. 2012; Davis et al. 2009; Henagan et al. 2014; Rayamajhi et al. 2013). For example, we have recently shown that dietary supplementation with a low dose of the quercetin, $50 \mathrm{ug} /$ day, creates a favorable acylcarnitine profile in skeletal muscle, suggesting an increased mitochondrial capacity to completely oxidize fatty acids through beta oxidation (Henagan et al. 2014). In addition, low but not high doses of quercetin supplementation attenuate diet-induced obesity and ameliorates HFD-induced whole body insulin resistance by increasing energy expenditure (Henagan et al. 2014).

Quercetin-rich foods that contain several forms of quercetin glycosides (Patil et al. 1995) may provide additional health benefits to dietary supplementation of purified quercetin aglycone alone, specifically with regard to improving insulin sensitivity (Jung et al. 2011). Red onions are an abundant source of flavonols, including high concentrations of all quercetin derivatives, such as quercetin aglycone and its glycosides (Patil et al. 1995). Red onions may be an effective treatment for hyperglycemia in diabetic patients (Eldin et al. 2010), with similar antidiabetic effects being noted in diabetic rats (Jung et al. 2011); however, these effects have not been directly attributed to quercetin (El-Demerdash et al. 2005; Kumari and Augusti 2002).

Given the beneficial effects of quercetin, we hypothesized that dietary supplementation with red onion extract (RO) would attenuate obesity and improve insulin sensitivity to a similar or greater extent than quercetin supplementation alone. We investigated whether the antiobesogenic and insulin-sensitizing effects of RO occur via upregulation of energy expenditure through a mechanism involving skeletal muscle mitochondrial biogenesis and function by administering purified dietary quercetin aglycone or RO containing quercetin aglycone and glycosides in a HFD to C57BL/6J mice for 9 weeks.

\section{Materials and methods}

Preparation and analysis of RO

Red onions were obtained from the local grocer, and a voucher specimen was prepared. The red onion $(\sim 1300 \mathrm{~g})$ was peeled and chopped, blended, and then extracted 1:4 (w/v) in $80 \%$ ethanol overnight. The slurry was filtered through Miracloth and centrifuged to remove particulates. Rotoevaporation was used to remove all of the ethanol. The remaining liquid was lyophilized to remove water and obtain $\sim 100 \mathrm{~g}$ of RO. The RO was hydrolyzed at $10 \mathrm{mg} /$ $\mathrm{mL}$ using $2.4 \mathrm{M} \mathrm{HCl}$ in $50 \%$ methanol at $80{ }^{\circ} \mathrm{C}$ for $2 \mathrm{~h}$ to determine the amount of quercetin aglycone equivalents. After hydrolysis, the samples (in triplicate) were centrifuged, filtered, and injected directly into HPLC-MS. Quercetin equivalents in the RO were determined using a standard curve prepared from purified quercetin.

Animals and diets

Five-weeks old C57BL/6J mice (Jackson Laboratories, Bar Harbor, MN, USA) were weaned onto low fat diet (LF; Research Diets 12450B, $10 \%$ kcal fat) for 1 weeks and then randomized into four separate treatment groups $(N=10$ /group): LF (Research Diets 12450B, $10 \% \mathrm{kcal}$ fat); high fat (HF; Research Diets D12451, $45 \%$ kcal fat); $\mathrm{HF}+\mathrm{Q}$ (Research Diets D08072305, $45 \%$ kcal fat with $17 \mathrm{mg} / \mathrm{kg}$ quercetin aglycone (Enzo Life Technologies ALX-385-001-G005; Farmingdale, NY, USA); or $\mathrm{HF}+\mathrm{RO}$ (Research Diets D08072306, $45 \% \mathrm{kcal}$ fat with $17 \mathrm{mg} / \mathrm{kg}$ of quercetin equivalents as RO). We have used formulated Research Diets (New Brunswick, NJ, USA) with quercetin previously and have shown that quercetin does not degrade in the diet, is absorbed into the systemic circulation, and is not toxic (Stewart et al. 2008). Food consumption and body weight and composition via nuclear magnetic resonance (Bruker Minispec, Billerica, MA, USA) were assessed weekly. After 9 weeks of feeding the respective diets, mice were killed and gastrocnemius or quadriceps muscles were used to make extracts for the measurement of fatty acid oxidation or frozen in liquid nitrogen for later analyses, respectively. Because specific fiber types may exhibit differential capacities for mitochondrial adaptation (Koves et al. 2008), whole tissue homogenates were used for all downstream experiments as a representative sample for the entire muscle group. All experiments were reviewed and approved by the Pennington Biomedical Research Center Institutional Animal Care and Use Committee, and the principles of laboratory animal care were followed.

Insulin sensitivity

After 7 weeks of feeding, whole body insulin sensitivity was assessed by glucose $(N=5 /$ group $)$ and insulin ( $N=5$ /group) tolerance tests. Mice were fasted overnight or for $4 \mathrm{~h}$ for GTT and ITT, respectively. All blood glucose measurements were obtained from the tail vein with a One 
Touch Basic Glucometer (Milpitas, CA, USA). For GTT, fasting blood glucose was measured, and mice were then injected intraperitoneally with $2 \mathrm{~g} / \mathrm{kg}$ body weight (BW) of glucose. Blood glucose concentrations were obtained 30 , 60 , and $120 \mathrm{~min}$ following the injection. For ITT, after measuring fasting blood glucose, mice were injected intraperitoneally with $0.75 \mathrm{U} / \mathrm{kg} \mathrm{BW}$ of insulin (NovolinR, Nordisk, Bagsvaerd, Denmark). Blood glucose measurements were made $15,30,60$, and $120 \mathrm{~min}$ following injection. Area under the curve (AUC) was calculated for individual mice using GraphPad Prism 5.0 and averaged for each treatment group.

\section{Indirect calorimetry}

Energy expenditure ( $N=6-8$ /group) was evaluated at 8 weeks after feeding the respective diets. Mice were housed individually in metabolic chambers (Oxymax Comprehensive Lab Animal System; Columbus Instruments, Columbus, OH, USA) on a 12-h light-dark cycle. After a 48-h acclimation period, the volume of oxygen consumption $\left(\mathrm{VO}_{2}\right)$ and volume of carbon dioxide production $\left(\mathrm{VCO}_{2}\right)$ were measured for $72 \mathrm{~h}$. Respiratory exchange ratio (RER) was calculated from $\mathrm{VCO}_{2}$ and $\mathrm{VO}_{2}$. Energy expenditure was calculated from $\mathrm{VO}_{2}$ and RER measurements $\left[\mathrm{VO}_{2} \times(3.815+(1.23 \times \mathrm{RER})) \times 40.1868\right]$ and expressed as kJ per kg BW per hour. Ambulatory and total physical activity, measured by beam breaks, was also obtained during this time.

Gene expression and mitochondrial number

For gene expression, total RNA was extracted from quadriceps muscle tissue using Tri Reagent (Molecular Research Center, Cincinnati, OH, USA) followed by further purification with a RNeasy mini kit (Qiagen, Valencia, CA, USA). The quantity and quality of the RNA was analyzed by spectrophotometry (ND-1000, NanoDrop Technologies, Wilmington, DE, USA). RNA was reverse transcribed into a cDNA library using M-MLV reverse transcriptase (Promega, Madison, WI, USA). qRT-PCR was performed using specific primers targeted towards exon-exon junctions that were designed using PrimerExpress v2.0.0 software (Applied Biosystems, Foster City, CA, USA). All samples were run in duplicate on the ABI 7900HT platform (Applied Biosystems, Foster City, CA, USA) using SyBR Green MasterMix (Applied Biosystems, Foster City, CA, USA). Gene expression was analyzed using a standard curve and normalization to cyclophilin B as the endogenous control.

Mitochondrial number was determined as previously described (Chang et al. 2012). For mitochondrial number, genomic DNA was isolated using a DNeasy kit per the manufacturer's protocol (Qiagen, Valencia, CA, USA). Genomic DNA was then quantified using SyBR Green qRT-PCR on the ABI 7900HT platform (Applied Biosystems, Foster City, CA, USA) with primers specific to a nuclear gene, lipoprotein lipase, 5'-GGATGGACGGTAA GAGTGATTC-3' (forward) and 5'-ATCCAAGGGTAGC AGACAGGT-3' (reverse), and primers specific to a mitochondrial gene, NADH dehydrogenase subunit I, 5'CCCATTCGCGTTATTCTT-3' (forward), and 5'-AAG TTGATCGTAACGGAAGC-3' (reverse). Mitochondrial number was determined as the relative expression of mitochondrial gene to nuclear gene using the delta delta $\mathrm{Ct}$ method.

Determination of incomplete beta oxidation

Tissue homogenates were prepared using mixed gastrocnemius skeletal muscle as previously described (Noland et al. 2007). Incomplete fatty acid oxidation rates were measured as the capture of ${ }^{14} \mathrm{C}$-labeled acid soluble metabolites (ASMs) from $\left[1-{ }^{14} \mathrm{C}\right]$ palmitate $(200 \mu \mathrm{M})$ as formerly shown (Noland et al. 2009a).

Statistical analysis

The data were analyzed with GraphPad Prism 5.0 statistical analysis software. Results are expressed as mean \pm standard error. Body composition parameters and GTT and ITT were analyzed by repeated measures ANOVA. All other measurements were analyzed by one-way ANOVA. A Tukey test was used post hoc as necessary. A $P$ value $<0.05$ was used to determine significance.

\section{Results}

\section{Development of RO}

A RO was created that contained several quercetin species, including the predominant quercetin aglycone. Quercitindiglycoside, quercitin-3-glucuronide, and quercitin-3-glucoside were also present in the RO; yet, the amounts were too small to contribute significantly to the pool of quercetin equivalents. From analysis of the hydrolyzed extract, using a standard curve prepared from pure quercetin, $2.2 \mathrm{mg} / \mathrm{L}$ of quercetin aglycone was measured in the sample which corresponds to $0.22 \%$ free quercetin equivalents in extract (Fig. 1a). Quercetin equivalents were determined to occur at approximately $21 \mathrm{mg} / \mathrm{g}$ in the RO (Fig. 1a).

We created a customized HFD in which the quercetin equivalents from $\mathrm{RO}(\mathrm{HF}+\mathrm{RO})$ were equal to that found in the $\mathrm{HF}+\mathrm{Q}$ diet (i.e., quercetin equivalents in the $\mathrm{HF}+\mathrm{RO}$ diet are at $17 \mathrm{mg} / \mathrm{kg}$ of diet), similar to the 
Fig. 1 a Quercetin equivalents were determined in $10 \mathrm{mg} / \mathrm{mL}$ of hydrolyzed RO by HPLCMS. HPLC-MS results are shown in triplicate. b Food intake was measured weekly over a $48 \mathrm{~h}$ period and is reported as $24 \mathrm{~h}$ mean \pm SEM c and as $24 \mathrm{~h}$ mean \pm SEM of caloric intake per gram of BW in mice fed a LFD (LF; white), HFD (HF; black), HFD plus $50 \mathrm{ug} /$ day quercetin $(\mathrm{HF}+\mathrm{Q}$; blue) or a HFD plus RO $(\mathrm{HF}+\mathrm{RO} ;$ red $)$

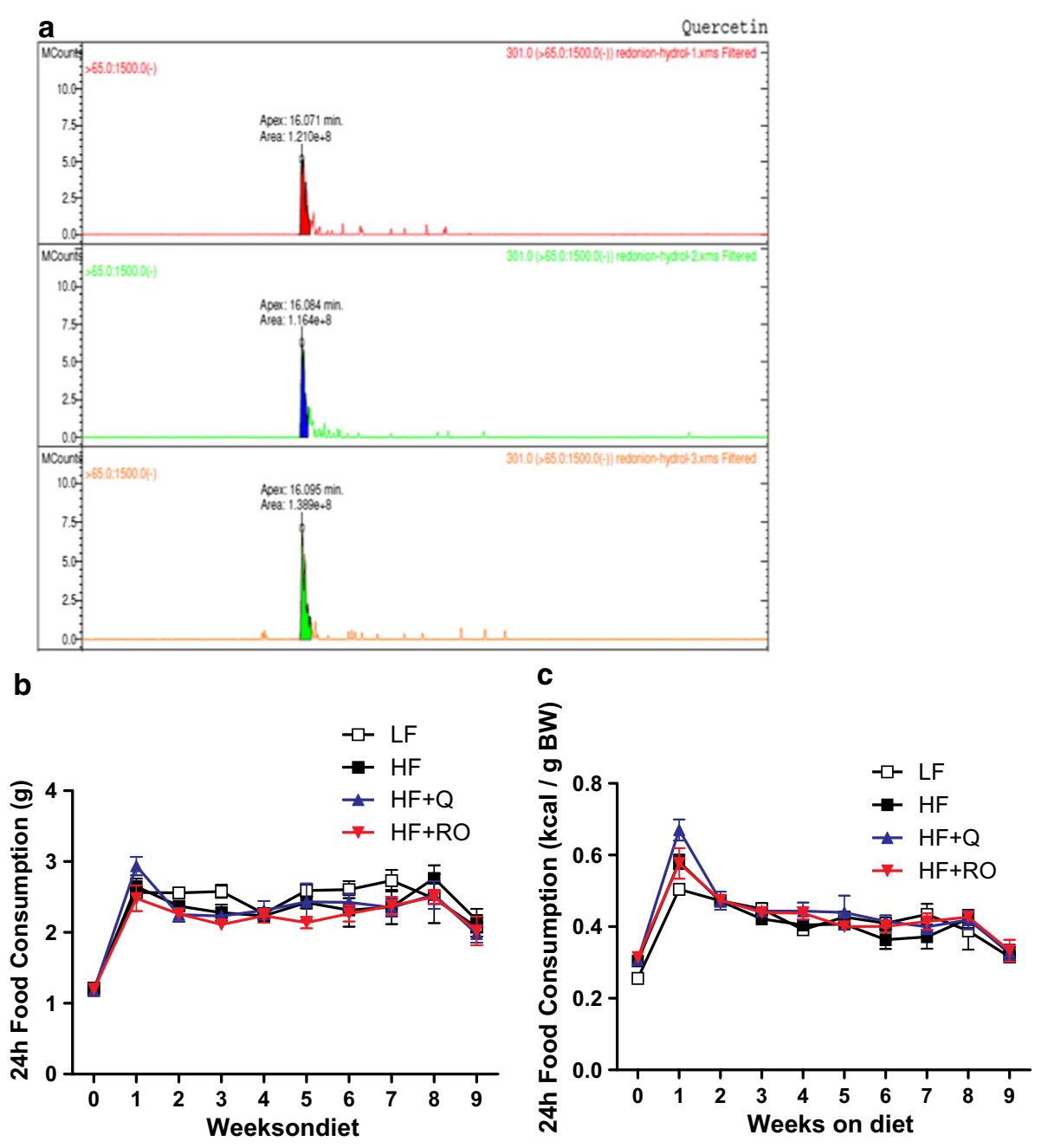

$\mathrm{HF}+\mathrm{Q}$ diet used in our previous study (Henagan et al. 2014). To rule out the possibility that the insulin-sensitizing effects of dietary quercetin and possible effects of RO were not due to decreases in caloric intake, we measured food consumption on a weekly basis and found that there were no differences in food or caloric intake between any groups (Fig. 1b, c). Based on the daily food intake, mice in $\mathrm{HF}+\mathrm{Q}$ and $\mathrm{HF}+\mathrm{RO}$ groups received $\sim 50 \mathrm{ug}$ of quercetin or quercetin equivalents/g of $\mathrm{BW}$ daily.

Quercetin- and RO-induced phenotypes

We observed a significant increase in BW in HF compared to LF (Fig. 2a). Both quercetin and RO supplementation attenuated the HFD-induced increase in BW, although the $\mathrm{BWs}$ of $\mathrm{HF}+\mathrm{Q}$ and $\mathrm{HF}+\mathrm{RO}$ were still significantly higher than that seen in LF (Fig. 2a). The increase in BW in HF compared to LF was due to a significant increase in adiposity in these animals (Fig. 2b, c), whereas decreased fat accumulation and body fat percentage was observed in
$\mathrm{HF}+\mathrm{Q}$ and $\mathrm{HF}+\mathrm{RO}$ compared to $\mathrm{HF}$ but increased compared to LF (Fig. 2b, c). Muscle mass was increased in all groups compared to LF (Fig. 2d). There was no difference in muscle mass between $\mathrm{HF}$ and $\mathrm{HF}+\mathrm{Q}$, although there was a decrease in $\mathrm{HF}+\mathrm{RO}$ compared to $\mathrm{HF}$ (Fig. 2d). However, when expressed as a percentage of body weight, percent muscle mass was decreased in all groups compared to $\mathrm{LF}$ and increased in $\mathrm{HF}+\mathrm{Q}$ and $\mathrm{HF}+\mathrm{RO}$ compared to HF (Fig. 2e).

Insulin sensitivity and metabolic effects

To assess insulin resistance in the animals, insulin (ITT) and glucose tolerance tests (GTT) were performed after 7 weeks on the respective diets. ITTs revealed that HF had significantly higher blood glucose concentrations compared to LF (Fig. 3a, c). This increase was abolished in $\mathrm{HF}+\mathrm{Q}$ and $\mathrm{HF}+\mathrm{RO}$ groups, such that blood glucose in these groups was similar to that of the LF mice (Fig. 3a, c). GTTs also showed impaired glucose tolerance that led to a 
Fig. 2 Body weight (a) and composition (b-e) were assessed weekly in LF (white), $\mathrm{HF}$ (black), $\mathrm{HF}+\mathrm{Q}$ (blue), and $\mathrm{HF}+\mathrm{RO}($ red). Body composition is reported as fat mass (b) percent fat mass

(c) muscle mass (d) and percent muscle mass (e). All values are reported as mean \pm SEM.

Significant differences between groups were determined by a repeated measured ANOVA and post hoc Tukey test. Differing superscripts represent significant differences at $P<0.05$

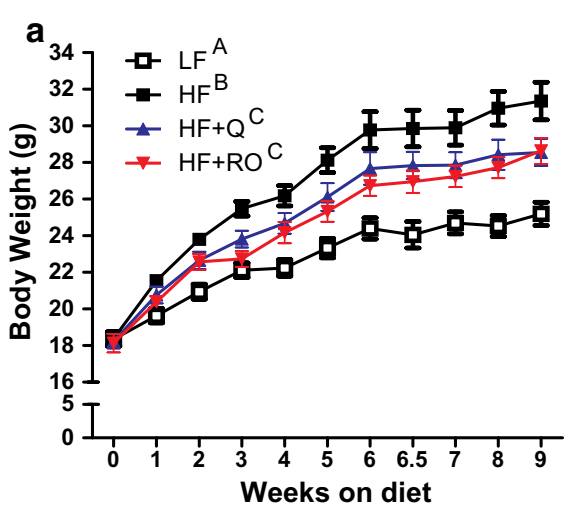

b
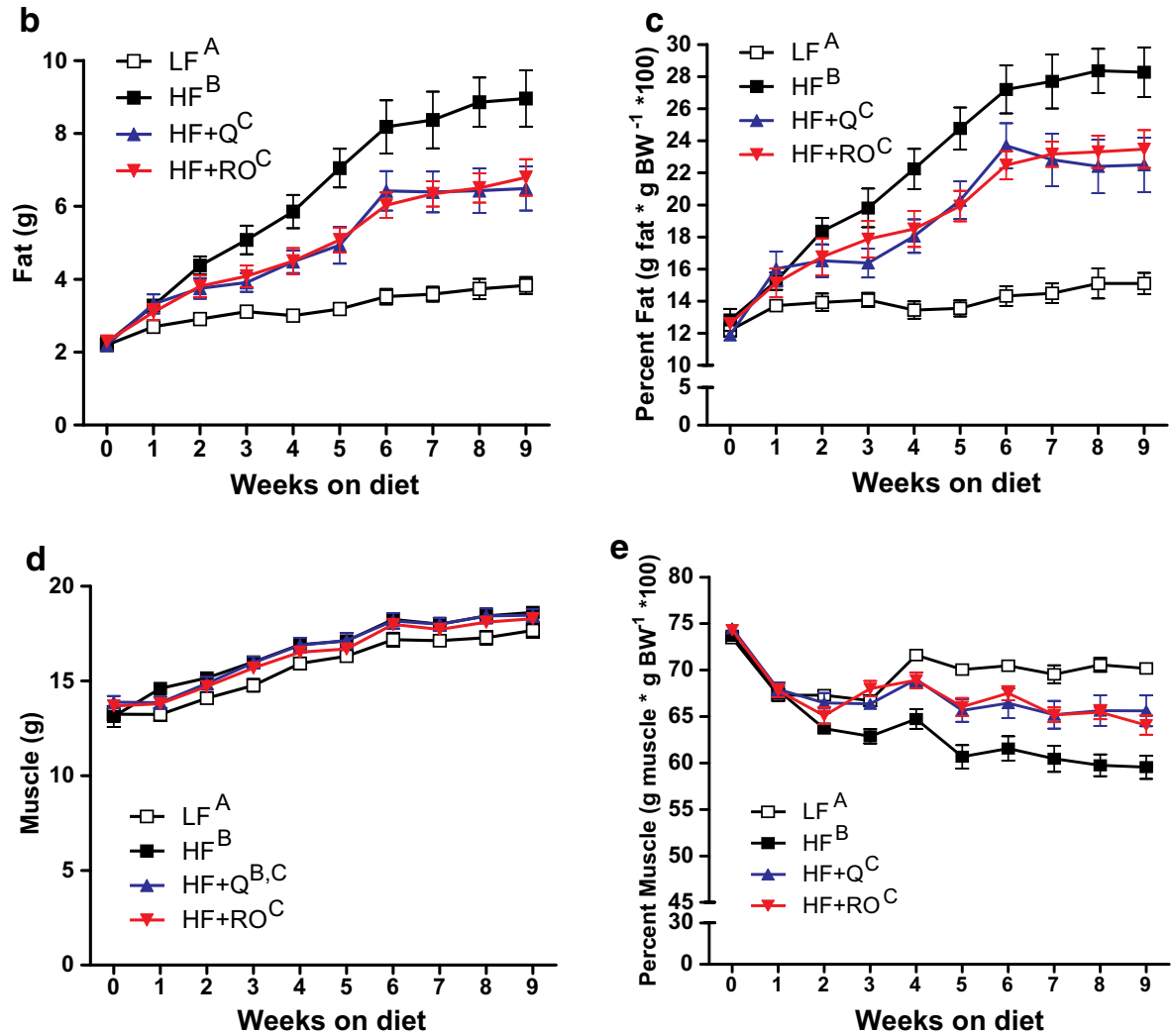

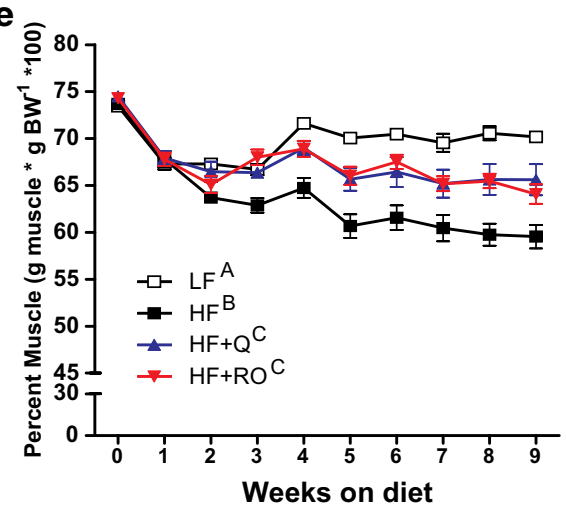

significant increase in blood glucose concentrations in $\mathrm{HF}$ compared to LF at the 60 min time point (Fig. 3b, d). Quercetin supplementation significantly decreased blood glucose at this time point compared to HF (Fig. 3b). RO tended to decrease blood glucose compared to $\mathrm{HF}$ at 60 min during the GTT $(P=0.08)$ (Fig. 3b).

At 8 weeks of feeding, mice were placed in metabolic chambers to assess energy expenditure, RER, and physical activity levels. $\mathrm{LF}, \mathrm{HF}+\mathrm{Q}$, and $\mathrm{HF}+\mathrm{RO}$ exhibited increased energy expenditure compared to $\mathrm{HF}$ (Fig. 4a). Interestingly, RO increased energy expenditure to a greater extent than quercetin supplementation alone in comparison to HF (Fig. 4a), although this increase was not significantly different. The observed increases in energy expenditure in
$\mathrm{LF}, \mathrm{HF}+\mathrm{Q}$, and $\mathrm{HF}+\mathrm{RO}$ were present in both the day (fasted) and night (fed) states (Fig. 4b). Total activity levels were also significantly increased in LF and $\mathrm{HF}+\mathrm{Q}$ compared to $\mathrm{HF}$ (Fig. 4b). Interestingly, total activity levels in $\mathrm{HF}+\mathrm{RO}$ were not different from HF (Fig. 4c). These data suggest that the mechanism contributing to increases in energy expenditure in $\mathrm{HF}+\mathrm{Q}$ versus $\mathrm{HF}+\mathrm{RO}$ may be different, as increases in $\mathrm{HF}+\mathrm{Q}$ but not $\mathrm{HF}+\mathrm{RO}$ are partially due to increases in total activity levels, similar to that observed in LF.

To determine whether quercetin or RO increase insulin sensitivity by switching substrate utilization or improving metabolic flexibility, RER was determined. RER was decreased in HF compared to LF, with similar decreases 
Fig. 3 Insulin sensitivity was assessed by insulin (ITT) and glucose (GTT) tolerance tests. Blood glucose measurements are shown as mean \pm SEM for ITT in (a) and for GTT in (b). Area under the curve (AUC) was calculated for each test and is presented as mean \pm SEM for the ITT in (c) and GTT in (d). Significant differences were determined by repeated measures ANOVA for ITT and GTT and one-way ANOVA for AUC. Post hoc Tukey tests were performed and differing superscripts represent significant differences at $P<0.05$
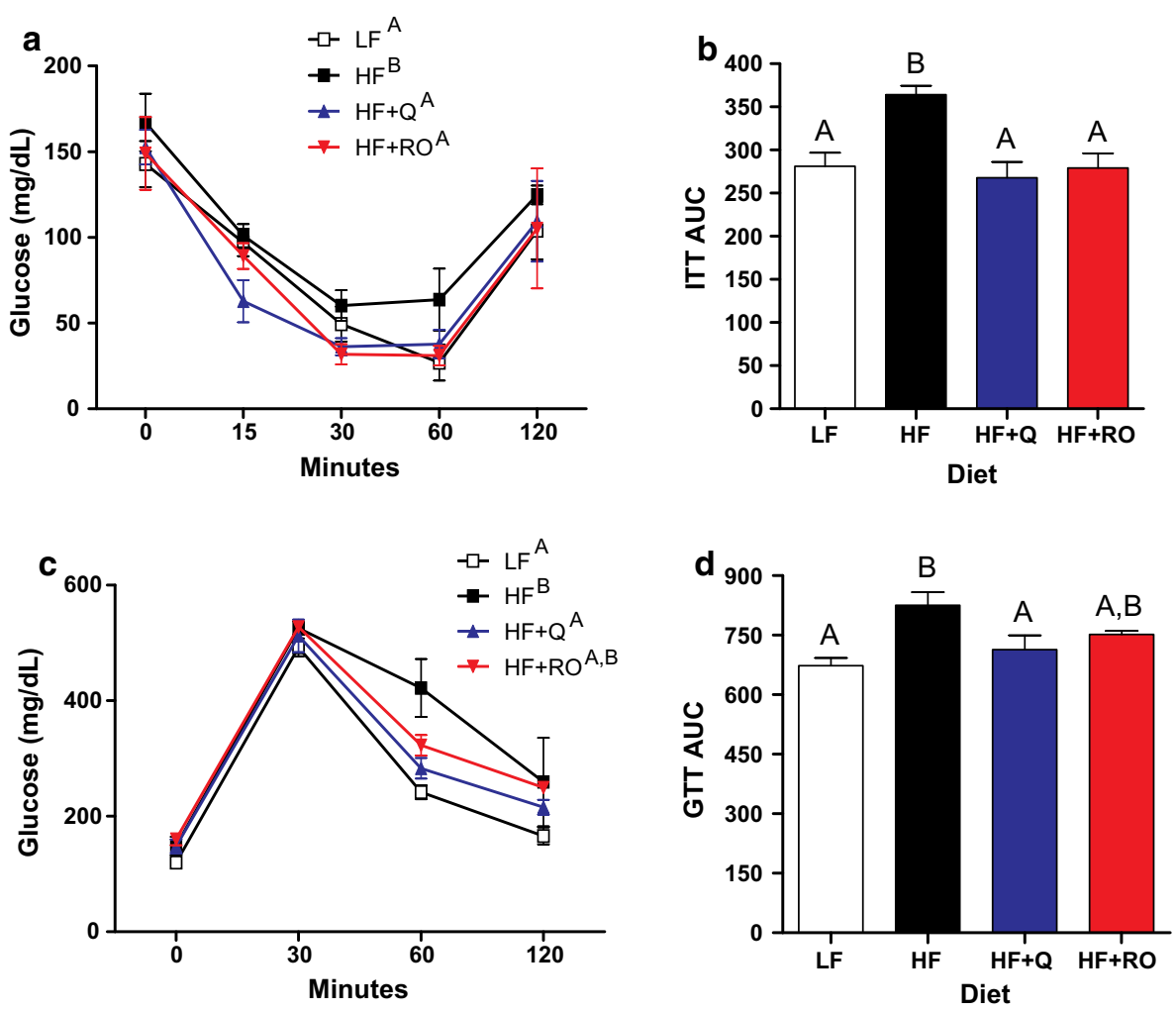

observed in HF + Q and HF + RO (Fig. 4d). Similarly, there were no statistical differences in RER between day and night periods in any group (Fig. 4e), indicating that the beneficial effects of dietary supplementation with either quercetin or RO were not due to improvements in metabolic flexibility. Thus, quercetin and RO act in a similar manner to increase whole body insulin sensitivity and energy expenditure without impacting substrate utilization and metabolic flexibility.

Quercetin- and RO-induced mitochondrial biogenesis and function

We have previously shown that a low dose of dietary quercetin supplementation increases $P g c l \alpha$ gene expression and improves the skeletal muscle acylcarnitine profile, suggesting beneficial mitochondrial adaptations (Henagan et al. 2014). To determine whether RO may act in a similar manner to induce mitochondrial adaptations that contribute to improvements in insulin sensitivity, we measured skeletal muscle mitochondrial number and function. There were no differences in mitochondrial number between LF and HF (Fig. 5a); however, both HF + Q and HF + RO showed significant increases in mitochondrial number compared to HF $(P=0.03)$. Furthermore, $\mathrm{HF}+\mathrm{Q}$ but not $\mathrm{HF}+\mathrm{RO}$ tended to increase mitochondrial number compared to $\mathrm{LF}$ (LF vs. $\mathrm{HF}+\mathrm{Q}, P=0.07$; $\mathrm{LF}$ vs. $\mathrm{HF}+\mathrm{RO}$, $P=0.10$ ) (Fig. 5a).
HFD has been shown to have varying effects on mitochondrial biogenesis that may occur independently of differences in mitochondrial function and insulin sensitivity and may be dependent on the duration of feeding and type and level of dietary fat (Hancock et al. 2008; Holloszy 2013). Thus, to test whether the HF + Q and HF + RO changes in mitochondrial number were associated with alterations in mitochondrial function, we assessed incomplete palmitate oxidation by measuring acid soluble metabolite (ASM) production in skeletal muscle homogenates. Consistent with previous reports (Noland et al. 2009b), increased incomplete fatty acid oxidation was observed in HF compared to LF-fed mice (Fig. 5b), which is suggestive of mitochondrial lipid overload in the HF group. Interestingly, both $\mathrm{HF}+\mathrm{Q}$ and $\mathrm{HF}+\mathrm{RO}$ groups exhibited ASM production at similar rates as HF-fed mice (Fig. 5b). However, it must be noted that the increased incomplete palmitate oxidation rates in the $\mathrm{HF}+\mathrm{Q}$ and $\mathrm{HF}+\mathrm{RO}$ groups are likely secondary to the increase in mitochondrial content. In support, normalization of ASMs to mitochondrial content (mtDNA) revealed that incomplete oxidation was still heightened in HF-fed mice, but returned to levels similar to LF-fed controls in response to both quercetin and red onion-supplemented mice. $\mathrm{CO}_{2}$ production, an indicator of fatty acid oxidation rate, was not different between any groups when expressed per tissue weight or per mitochondrial number (data not shown). Collectively, these data support the notion that expansion 

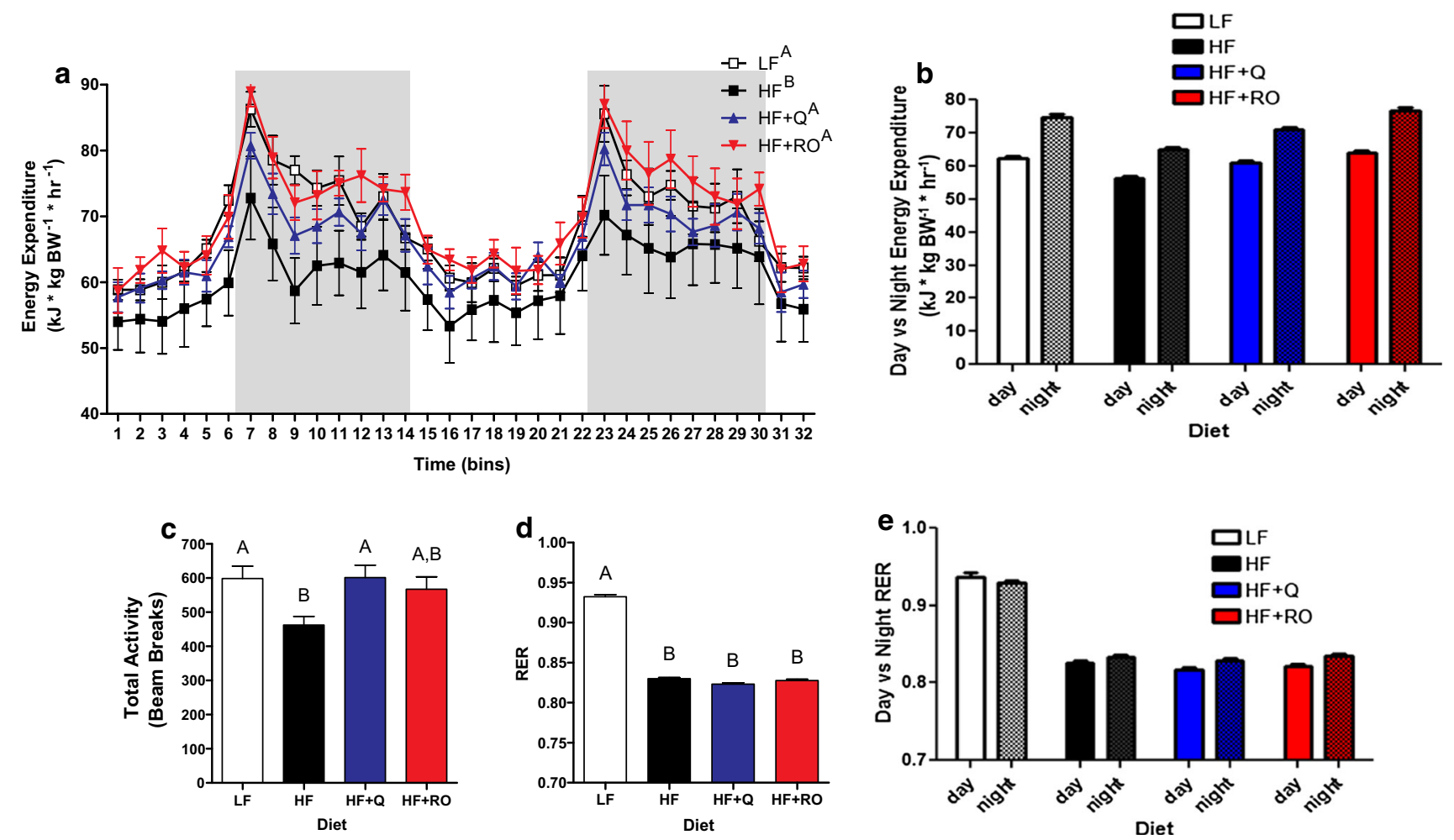

Fig. 4 Energy expenditure, physical activity, and respiratory exchange ratio (RER) were measured by indirect calorimetry in C57BL/6J mice after 8 weeks of feeding respective diets. a Energy expenditure was calculated from measured $\mathrm{VO}_{2}$ and RER and is shown respective to $\mathrm{BW}$ for each treatment group. Night periods are represented by gray bars. b Average day and night energy

of mitochondrial content in $\mathrm{HF}+\mathrm{Q}$ and $\mathrm{HF}+\mathrm{RO}$ groups is sufficient to allow these mice to catabolize a similar amount of lipid as the HF mice, while also limiting the deleterious effects of mitochondrial lipid overload.

Quercetin- and RO-induced changes in mitochondrial gene expression

Previous reports have shown that mRNA levels of mitochondrial-encoded oxidative phosphorylation (OXPHOS) genes may serve as markers of mitochondrial function, and their expression is decreased in skeletal muscle of diabetic individuals (Asmann et al. 2006). To determine whether quercetin and RO may increase mitochondrial number and function through a mechanism related to gene transcription of mtDNA, transcript levels of the 13 mitochondrialencoded genes that give rise to polypeptides contributing to the subunits of the respiratory chain were measured. After 9 weeks of feeding, $\mathrm{HF}+\mathrm{Q}$ exhibited lower $\mathrm{NADH}$ dehydrogenase $(\mathrm{Nd}) 3(\mathrm{Nd} 3)$ and $\mathrm{Nd} 4$, cyclooxygenase (Cox) 1 (Coxl), and adenosine triphosphate (Atp) 8 (Atp8) mRNA levels compared to HF; $N d l$ also tended to decrease $(P=0.07) \quad$ (Fig. 6). Additionally, $\mathrm{HF}+\mathrm{Q}$ exhibited expenditure values are shown for each treatment group. c Physical activity levels and RER (d) are shown as the average over a 3 days period. d Average day and night RER values are shown for each treatment group. All data are presented as mean \pm SEM. Significant differences between groups by one-way ANOVA and post hoc Tukey are denoted by differing superscripts

decreases in mRNA levels of $N d 4 l, N d 5, \operatorname{Cox} 1, \operatorname{Cox} 2$, Cox3, Atp6, and Atp8 compared to LF (Fig. 6). HF + RO showed decreased mRNA levels of $N d 5$ and Coxl only in comparison to LF and no differences in mRNA levels compared to HF (Fig. 6). With the exception of the decreased transcript levels of $\mathrm{Nd} 5$ and Coxl in $\mathrm{HF}+\mathrm{Q}$ and $\mathrm{HF}+\mathrm{RO}$ when compared to LF (Fig. 6), there were no other similar trends in mRNA levels of mitochondrialencoded genes in response to quercetin and $\mathrm{RO}$ supplementation.

\section{Discussion}

Onion ingestion improves hyperglycemia in diabetic patients and has antidiabetic effects in mice (Eldin et al. 2010; Jung et al. 2011); yet, the specific bioactive component responsible for these health benefits is unknown. Red onions are rich in the bioflavonoid quercetin (Patil et al. 1995) and have pleotropic health benefits (Anhê et al. 2012; Eid et al. 2010; Henagan et al. 2014; Jeong et al. 2012; Stewart et al. 2008; Xu et al. 2013), making them an attractive candidate for use as dietary medicine. Here, we 
Fig. 5 a Mitochondrial number was determined as the amount of mitochondrial DNA relative to nuclear DNA and b incomplete beta oxidation was determined by measuring acid soluble metabolites (ASM) produced from palmitate oxidation in skeletal muscle samples. c Mitochondrial function in the form of incomplete beta oxidation was determined as the average ASM per skeletal muscle mitochondrion. Mean \pm SEM shown. Significant differences between groups were determined by one way ANOVA and post hoc Tukey. Differing superscripts indicate significant differences between groups at $P<0.05$ a
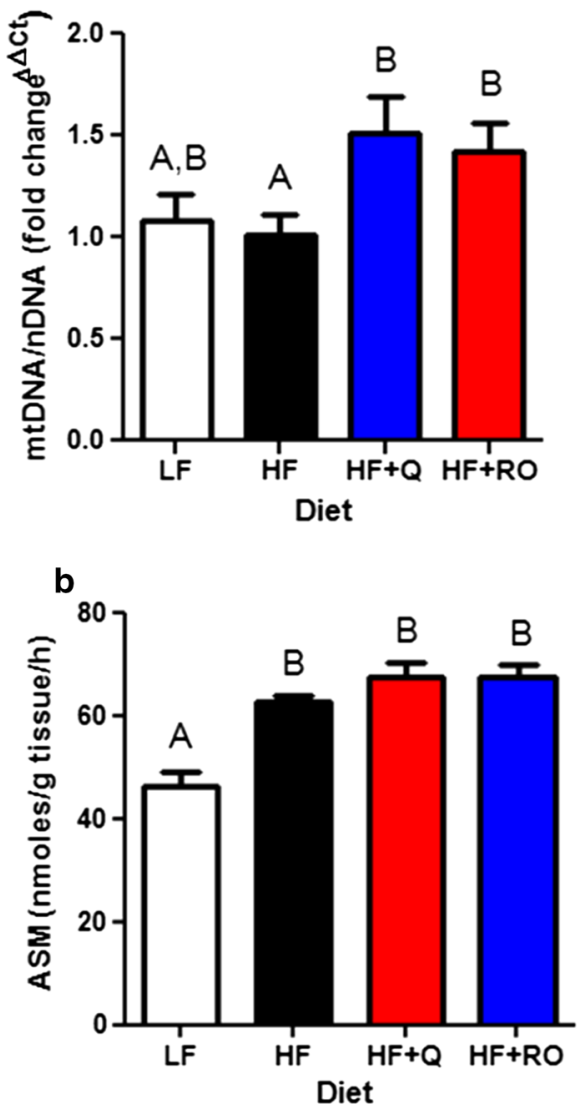

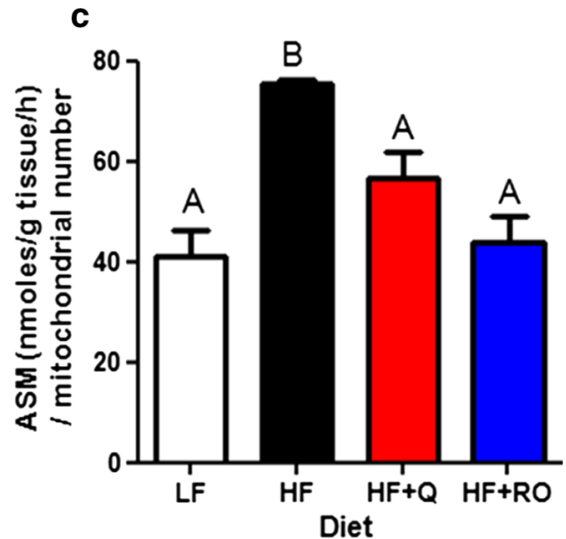

tested the hypothesis that quercetin and RO supplementation would similarly attenuate HFD-induced insulin resistance by limiting adiposity and increasing energy expenditure and that these changes would occur through a mechanism related to improvements in skeletal muscle mitochondrial number and function. The present results show that a low dose of dietary quercetin aglycone incorporated either as a purified compound or as component in RO can similarly attenuate obesity and HFD-induced insulin resistance in $\mathrm{C} 57 \mathrm{BL} / 6 \mathrm{~J}$ mice, suggesting that the major bioactive component of red onion may indeed be quercetin. Although we observed similar changes in energy expenditure and skeletal muscle mitochondrial adaptations in response to both purified quercetin aglycone and $\mathrm{RO}$, the fact that the results were not identical suggests that the molecular mechanism(s) at the level of the mitochondria may include points of differential regulation related to other compounds in RO, such as the quercetin glycosides.

The effectiveness of quercetin in treating obesity and insulin resistance is a topic of debate and may be attributed to the fact that quercetin's effects are dose- and timedependent (Egert et al. 2008; Henagan et al. 2014). For example, low, chronic doses of quercetin, such as that used in the present study, improve insulin sensitivity, while high or acute doses exacerbate HFD-induced insulin resistance (Henagan et al. 2014). In addition, food components alter quercetin absorption and bioavailability and further complicate the dose- and temporal-dependent effects of dietary quercetin supplementation (Guo et al. 2013). Given the possible health benefits of whole food extracts, it is possible that supplementation with a quercetin-rich RO that contains potentially synergistic compounds may serve as a better alternative for disease prevention and treatment (Hollman et al. 1995). Despite the contention between purified compounds versus extracts, we are able to show here that both treatments (quercetin or RO) result in lower BWs and HFD-induced gains in adiposity. These effects persisted throughout the study and confirm that $50 \mathrm{ug} /$ day quercetin is effective in attenuating HFD-induced weight gain with concomitant reductions in fat mass, similar to our previous report (Henagan et al. 2014). Additionally, similarities in the effectiveness of purified quercetin and RO in improving BW and adiposity suggest that quercetin may be the primary bioactive component within RO leading to these results.

Similar quercetin- and RO-induced decreases in BW and adiposity occurred in conjunction with increases in energy expenditure and no change in caloric intake. These results 

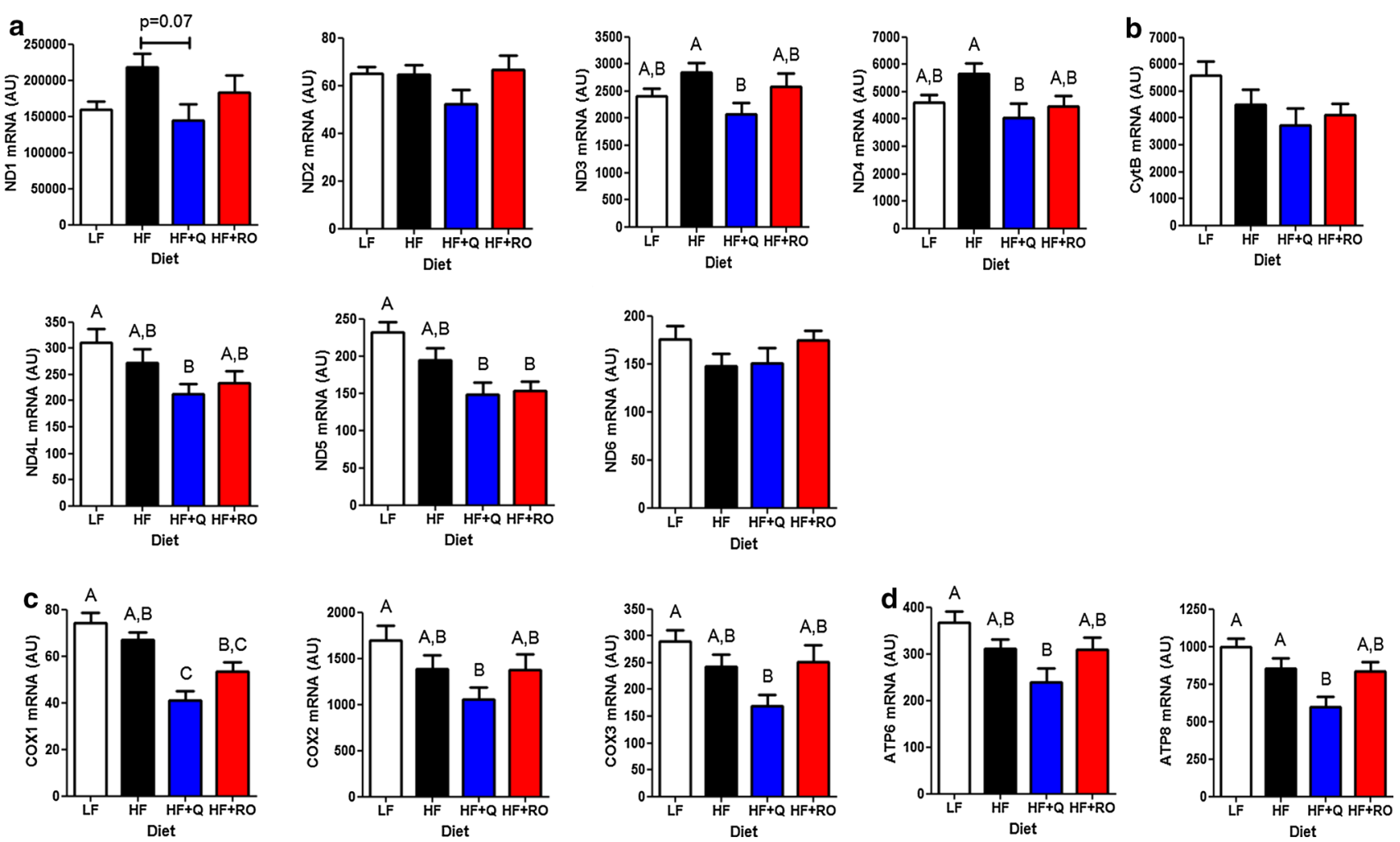

Fig. 6 mRNA levels of genes encoding polypeptides for subunits of the mitochondrial complex I (a) complex III (b) complex IV (c) and complex V (d) were determined by qRT-PCR using the standard curve method in skeletal muscle samples. Values are reported as mean \pm SEM in arbitrary units (AU). Significant differences between

groups were determined by one-way ANOVA and post hoc Tukey. Differing superscripts indicate significant differences between groups at $P<0.05$. ND NADH dehydrogenase, $C y t B$ cytochrome B, COX cyclooxygenase, and $A T P$ adenosine triphosphate

suggest that both quercetin and RO limit HFD-induced fat accumulation by increasing energy expenditure through increases in basal metabolic rate or physical activity levels without altering the thermogenic effect of food (Ravussin et al. 1986). Indeed, higher physical activity levels were observed in $\mathrm{HF}+\mathrm{Q}$ compared to $\mathrm{HF}$, indicating that quercetin-induced energy expenditure is partially related to increases in activity. Interestingly, $\mathrm{HF}+\mathrm{RO}$ did not exhibit significant increases in physical activity levels in comparison to $\mathrm{HF}$, indicating that RO-induced increases in energy expenditure may occur through a differential mechanism than that induced by quercetin.

Consistent with the similarities between $\mathrm{HF}+\mathrm{Q}$ and $\mathrm{HF}+\mathrm{RO}$ in body composition and energy expenditure, insulin sensitivity measured by ITT and GTT revealed the strong potential for these supplements to attenuate HFDinduced insulin resistance and restore levels to those similar to animals fed a LFD. These results are consistent with other studies that show that dietary quercetin supplementation ameliorates HFD-induced insulin resistance in a time-dependent manner (Henagan et al. 2014). Improvements in energy expenditure and insulin sensitivity are associated with beneficial skeletal muscle mitochondrial adaptations (Asmann et al. 2006; Carrasco-Pozo et al. 2012; Davis et al. 2009; Henagan et al. 2014; Rayamajhi et al. 2013; Sparks et al. 2005; Stewart et al. 2008). Specifically, previous studies revealed that elevated incomplete fatty acid oxidation, evidenced by differences in acylcarnitine profiles that show increases in long and medium chain acylcarnitines with respect to short chain acylcarnitines, is a common trait observed in insulin resistant skeletal muscle and strategies that diminished this mitochondrial lipid overload had insulin-sensitizing effects (Koves et al. 2008). In the present study, incomplete fatty acid oxidation was determined through measurement of ASM which represents the metabolic byproducts of mitochondrial and peroxisomal fatty acid oxidation, including incompletely oxidized medium chain fatty acids (Noland et al. 2007). The fact that quercetin and RO supplementation increased mitochondrial content to the extent where incomplete lipid oxidation per mitochondrion was normalized to LF controls suggests these botanicals effectively limit the lipid stress placed on the mitochondria, which could play a significant role in the insulin-sensitizing action of these botanicals. Although our results confirm previous reports that quercetin supplementation increases muscle 
mitochondrial number and improves mitochondrial function (Henagan et al. 2014), this is the first study to provide evidence that RO supplementation is as effective as supplementation as quercetin aglycone alone in preventing HFD-induced insulin resistance and has a similar impact on skeletal muscle mitochondrial biogenesis and lipid oxidation.

Quercetin-induced increases in mitochondrial number have been shown to occur in conjunction with increased time to exhaustion during exercise (Davis et al. 2009). Here, we found that quercetin supplementation led to increased skeletal muscle mitochondrial number and higher physical activity levels, suggesting that quercetin-induced skeletal muscle mitochondrial biogenesis may be the cause or the result of changes in physical activity leading to subsequent increases in energy expenditure. We observed a similar induction of energy expenditure, mitochondrial biogenesis, and normalization of incomplete fatty acid oxidation when expressed per mitochondrion in $\mathrm{HF}+\mathrm{RO}$ as in $\mathrm{HF}+\mathrm{Q}$; yet, $\mathrm{HF}+\mathrm{RO}$ did not show a change in physical activity. Thus, we speculate that specific quercetin and RO-induced mitochondrial adaptations may contribute differentially to regulate energy expenditure.

To further explore this possibility, we examined transcript levels of all 13 mitochondrial-encoded genes that are essential for proper respiratory chain function in skeletal muscle (Wallace 1999). These genes compromise subunits of complex I or NADH dehydrogenase, $N d 1, N d 2, N d 3$, $N d 4, N d 4 l, N d 5$, and $N d 6$, those of complex III or ubiquinal-cytochrome c reductase, cytochrome $B(C y t B)$, those of complex IV, or cytochrome c oxidase, Coxl, Cox2 and Cox3, and those in complex V or ATP synthase, Atp6 and Atp8. Similar significant decreases in mRNA levels of those genes encoding subunits for complexes IV and V but not in complexes I and III were found in HF + Q but not $\mathrm{HF}+\mathrm{RO}$ and may be indicative of alterations occurring systematically with biological relevance to the OXPHOS functioning (Wallace 1999). Quercetin is known to increase permeability of the mitochondrial membrane (De Marchi et al. 2009), a function that allows proton dissipation down the concentration gradient, abolishing the electrochemical gradient, and preventing coupling of oxidation from ATP production. Thus, quercetin-induced alterations in mtDNA-encoded transcript levels of IV and V but not I and III may indicate an increased ability for uncoupling of NADH and FADH oxidation to ATP generation to occur. The effects of quercetin and RO on the uncoupling potential in muscle have not been explored, but our data suggest that differences in the expression of mitochondrialencoded genes representing a break point in the electron transport chain may explain the differences in activitybased changes in energy expenditure between these two groups.
The current study has several strengths. First, while others have previously published an antidiabetic effect of onion supplementation, the mechanism for this adaptation remained elusive. Here, we show that RO increases skeletal muscle mitochondrial number and function, leading to increased energy expenditure. Second, the study determines whether the insulin sensitizing and antiobesogenic effects of red onion are more effective than purified quercetin aglycone. Other studies have independently determined the effectiveness of quercetin aglycone or red onion on insulin sensitivity and obesity, but no reports have compared the two. Direct comparison of the effects of quercetin aglycone and RO in the current study provides evidence that the main bioactive component of red onion may be quercetin. However, a weakness of the current study is the inability to directly determine whether other components of RO to contribute to its beneficial effects. Administering a RO deplete in quercetin may help to elucidate this issue in future studies. Third, we report that HFD does not alter mtDNA-ecoded gene transcript levels compared to animals fed a LFD. It has previously been shown that HFD in C57BL/6J mice induces insulin resistance in skeletal muscle by OXPHOS gene expression (Sparks et al. 2005) and that genes encoding OXPHOS proteins in skeletal muscle of type 2 diabetic individuals exhibit increases in transcript levels (Asmann et al. 2006). However, in both cases, the genes of interest were encoded only in the nuclear genome and mtDNA-encoded mitochondrial genes remained unexplored. In addition to these strengths, several weaknesses also exist in the present study. First, it should be noted that while quantifying mtDNA may serve as a surrogate measure of mitochondrial content (Casuso et al. 2013; Chang et al. 2012; Davis et al. 2009; Drew et al. 2014), it is not a direct measure of mitochondrial number and a recent study in humans shows that mtDNA does not correlate with other measures of mitochondrial number (Larsen et al. 2012). Second, while ASM represents incomplete beta oxidation and is used to assess differences in mitochondrial function in the present study, ASM may also be produced from incomplete beta oxidation of palmitate by peroxisomes (Noland et al. 2007). Thus, it is possible that the beneficial effects of quercetin and RO on the completeness of beta oxidation, as measured by ASM, may also partially be due to improvements in peroxisomal function.

To summarize, we demonstrate that a low dose of quercetin, both by itself or as a component in a RO, increases energy expenditure and results in improvements in body composition and insulin sensitivity. The benefits of purified quercetin aglycone or RO supplementation may be partially due to increases in skeletal muscle mitochondrial number which improve fat metabolism in a manner that limits mitochondrial lipid overload, and these changes may 
occur through differential regulation of mtDNA-encoded gene expression. The ability of quercetin or RO not only to prevent obesity or insulin resistance but also to reverse these metabolic derangements should be further explored in future studies.

Acknowledgments This work was funded by National Institutes of Health National Center for Complementary and Alternative Medicine (5P50-AT002776-09; TMH), National Institutes of Health National Institute of Diabetes and Digestive and Kidney Diseases (5T32DK064584-09; TMH), and National Institutes of Health Centers of Biomedical Research Excellence (8P20-GM103528-07; RCN). This work also used the Genomics Core Facility at PBRC which is partially supported by National Institutes of Health Centers of Biomedical Research Excellence (8P20-GM103528-07) and National Institutes of Health National Obesity Research Centers (5P30DK072476-07) center grants.

Conflict of interest The authors have no conflicts of interest to disclose.

Ethical standard All institutional and national guidelines for the care and use of laboratory animals were followed.

\section{References}

Anhê GF, Okamoto MM, Kinote A, Sollon C, Lellis-Santos C, Anhê FF, Lima GA, Hirabara SM, Velloso LA, Bordin S et al (2012) Quercetin decreases inflammatory response and increases insulin action in skeletal muscle of ob/ob mice and in L6 myotubes. Eur J Pharmacol 689:285-293

Asmann YW, Stump CS, Short KR, Coenen-Schimke JM, Guo Z, Bigelow ML, Nair KS (2006) Skeletal muscle mitochondrial functions, mitochondrial dna copy numbers, and gene transcript profiles in type 2 diabetic and nondiabetic subjects at equal levels of low or high insulin and euglycemia. Diabetes 55:3309-3319

Carrasco-Pozo C, Mizgier ML, Speisky H, Gotteland M (2012) Differential protective effects of quercetin, resveratrol, rutin and epigallocatechin gallate against mitochondrial dysfunction induced by indomethacin in Caco- 2 cells. Chem Biol Interact 195:199-205

Casuso RA, Martínez-López EJ, Nordsborg NB, Hita-Contreras F, Martínez-Romero R, Cañuelo A, Martínez-Amat A (2013) Oral quercetin supplementation hampers skeletal muscle adaptations in response to exercise training. Scand J Med Sci Sports 24:920-927

Chang JS, Fernand V, Zhang Y, Shin J, Jun H-J, Joshi Y, Gettys TW (2012) NT-PGC-1 $\alpha$ protein is sufficient to link $\beta 3$-adrenergic receptor activation to transcriptional and physiological components of adaptive thermogenesis. J Biol Chem 287:9100-9111

Davis JM, Murphy EA, Carmichael MD, Davis B (2009) Quercetin increases brain and muscle mitochondrial biogenesis and exercise tolerance. Am J Physiol Regulat Integr Comp Physiol 296:R1071-R1077

De Marchi U, Biasutto L, Garbisa S, Toninello A, Zoratti M (2009) Quercetin can act either as an inhibitor or an inducer of the mitochondrial permeability transition pore: a demonstration of the ambivalent redox character of polyphenols. Biochim Biophys Acta (BBA) Bioenerg 1787:1425-1432

Drew BG, Ribas V, Le JA, Henstridge DC, Phun J, Zhou Z, Soleymani T, Daraei P, Sitz D, Vergnes L et al (2014) HSP72 Is a mitochondrial stress sensor critical for Parkin action, oxidative metabolism, and insulin sensitivity in skeletal muscle. Diabetes 63:1488-1505
Egert S, Wolffram S, Bosy-Westphal A, Boesch-Saadatmandi C, Wagner AE, Frank J, Rimbach G, Mueller MJ (2008) Daily quercetin supplementation dose-dependently increases plasma quercetin concentrations in healthy humans. J Nutr 138:1615-1621

Eid HM, Martineau LC, Saleem A, Muhammad A, Vallerand D, Benhaddou-Andaloussi A, Nistor L, Afshar A, Arnason JT, Haddad PS (2010) Stimulation of AMP-activated protein kinase and enhancement of basal glucose uptake in muscle cells by quercetin and quercetin glycosides, active principles of the antidiabetic medicinal plant Vaccinium vitis-idaea. Mol Nutr Food Res 54:991-1003

El-Demerdash FM, Yousef MI, El-Naga NI (2005) Biochemical study on the hypoglycemic effects of onion and garlic in alloxaninduced diabetic rats. Food Chem Toxicol 43:57-63

Eldin IMT, Ahmed EM, Elwahab HMA (2010) Preliminary study of the clinical hypoglycemic effects of Allium cepa (red onion) in type 1 and type 2 diabetic patients. Environ Health Insights 4:71-77

Guo Y, Mah E, Davis CG, Jalili T, Ferruzzi MG, Chun OK, Bruno RS (2013) Dietary fat increases quercetin bioavailability in overweight adults. Mol Nutr Food Res 57:896-905

Hancock CR, Han D-H, Chen M, Terada S, Yasuda T, Wright DC, Holloszy JO (2008) High-fat diets cause insulin resistance despite an increase in muscle mitochondria. Proc Natl Acad Sci 105:7815-7820

Henagan TM, Lenard NR, Gettys TW, Stewart LK (2014) Dietary quercetin supplementation in mice increases skeletal muscle $P G C 1 \propto$ expression, improves mitochondrial function and attenuates insulin resistance in a time-specific manner. PLoS One 9:e89365

Hollman PC, de Vries JH, van Leeuwen SD, Mengelers MJ, Katan MB (1995) Absorption of dietary quercetin glycosides and quercetin in healthy ileostomy volunteers. Am J Clin Nutr 62:1276-1282

Holloszy JO (2013) "Deficiency" of mitochondria in muscle does not cause insulin resistance. Diabetes 62:1036-1040

Jeong S-M, Kang M-J, Choi H-N, Kim J-H, Kim J-I (2012) Quercetin ameliorates hyperglycemia and dyslipidemia and improves antioxidant status in type 2 diabetic $\mathrm{db} / \mathrm{db}$ mice. Nutr Res Pract 6:201-207

Jia H, Aw W, Egashira K, Takahashi S, Aoyama S, Saito K, Kishimoto Y, Kato H (2014) Coffee intake mitigated inflammation and obesity-induced insulin resistance in skeletal muscle of high-fat diet-induced obese mice. Genes Nutr 9:1-10

Jung JY, Lim Y, Moon MS, Kim JY, Kwon O (2011) Onion peel extracts ameliorate hyperglycemia and insulin resistance in high fat diet/streptozotocin-induced diabetic rats. Nutr Metabol 8:18

Koves TR, Ussher JR, Noland RC, Slentz D, Mosedale M, Ilkayeva O, Bain J, Stevens R, Dyck JRB, Newgard CB et al (2008) Mitochondrial overload and incomplete fatty acid oxidation contribute to skeletal muscle insulin resistance. Cell Metab 7:45-56

Kumari K, Augusti KT (2002) Antidiabetic and antioxidant effects of $\mathrm{S}$-methyl cysteine sulfoxide isolated from onions (Allium cepa Linn) as compared to standard drugs in alloxan diabetic rats. Indian J Exp Biol 40:1005-1009

Larsen S, Nielsen J, Hansen CN, Nielsen LB, Wibrand F, Stride N, Schroder HD, Boushel R, Helge JW, Dela F et al (2012) Biomarkers of mitochondrial content in skeletal muscle of healthy young human subjects. J Physiol 590:3349-3360

Lowell BB, Shulman GI (2005) Mitochondrial dysfunction and type 2 diabetes. Science 307:384-387

Noland RC, Woodlief TL, Whitfield BR, Manning SM, Evans JR, Dudek RW, Lust RM, Cortright RN (2007) Peroxisomalmitochondrial oxidation in a rodent model of obesity-associated 
insulin resistance. Am J Physiol Endocrinol Metab 293:E986E1001

Noland RC, Koves TR, Seiler SE, Lum H, Lust RM, Ilkayeva O, Stevens RD, Hegardt FG, Muoio DM (2009a) Carnitine insufficiency caused by aging and overnutrition compromises mitochondrial performance and metabolic control. J Biol Chem 284:22840-22852

Noland RC, Koves TR, Seiler SE, Lum H, Lust RM, Ilkayeva O, Stevens RD, Hegardt FG, Muoio DM (2009b) Carnitine insufficiency caused by aging and overnutrition compromises mitochondrial performance and metabolic control. J Biol Chem 284:22840-22852

Patil BS, Pike LM, Yoo KS (1995) Variation in the quercetin content in different colored onions (Allium cepa L.). J Am Soc Hortic Sci 120:909-913

Ravussin E, Lillioja S, Anderson T, Christin L, Bogardus C (1986) Determinants of 24-hour energy expenditure in man. Methods and results using a respiratory chamber. J Clin Investig 78:1568-1578

Rayamajhi N, Kim S-K, Go H, Joe Y, Callaway Z, Kang J-G, Ryter SW, Chung HT (2013) Quercetin induces mitochondrial biogenesis through activation of HO-1 in HepG2 cells. Oxid Med Cell Longev 2013:10

Sparks LM, Xie H, Koza RA, Mynatt R, Hulver MW, Bray GA, Smith SR (2005) A high-fat diet coordinately downregulates genes required for mitochondrial oxidative phosphorylation in skeletal muscle. Diabetes 54:1926-1933

Stewart LK, Soileau JL, Ribnicky D, Wang ZQ, Raskin I, Poulev A, Majewski M, Cefalu WT, Gettys TW (2008) Quercetin transiently increases energy expenditure but persistently decreases circulating markers of inflammation in C57BL/6J mice fed a high-fat diet. Metabolism 57:S39-S46

Szendroedi J, Phielix E, Roden M (2012) The role of mitochondria in insulin resistance and type 2 diabetes mellitus. Nat Rev Endocrinol 8:92-103

Wallace DC (1999) Mitochondrial diseases in man and mouse. Science 283:1482-1488

Xu M, Hu J, Zhao W, Gao X, Jiang C, Liu K, Liu B, Huang F (2013) Quercetin differently regulates insulin-mediated glucose transporter 4 translocation under basal and inflammatory conditions in adipocytes. Mol Nutr Food Res 58:931-941 